www.jmscr.igmpublication.org

Index Copernicus Value: 79.54

ISSN (e)-2347-176x ISSN (p) 2455-0450

crossrefDOI: https://dx.doi.org/10.18535/jmscr/v7i3.203

Journal Of Medical Science And Clinical Research

\title{
Neuroimaging Findings in Patients Presenting with Seizure to a Tertiary Care Hospital: A Cross Sectional Study
}

\author{
Authors \\ Dr Soumya Ranjan Pradhan, Dr Karishma Parida, Dr P C Karua, Dr B K Kullu \\ VIMSAR
}

\begin{abstract}
Introduction: In Indian medical practice assessment of patients presenting with seizure is a challenge for a physician in evaluating and treating them. After introduction of neuroimaging in seizure, they have been a great boon both for diagnosis of cerebral lesions as well as clinical management of patients with neurological impairments.

Objective: To study the neuroimaging findings in patients presenting with seizure and to determine etiology and to correlate type of seizure with neuroimaging findings.

Study Design: Hospital based cross sectional study

Study Setting: This study was done in department of Internal medicine, VIMSAR, ODISHA

Study Duration: Study duration was from October 2016 to September 2018 (24 months)

Subjects and Methods: Total 212 cases of age > 14 years who presented with seizure were studied and patients having implants, pregnant and claustrophobic patients were excluded. All the patients were subjected to CT scan and MRI of brain by a 160 slice CT scan machine and 1.5 tesla MRI scanner respectively. Various details of neuroimaging findings were studied like- Any lesion present, signal intensity/density, surrounding edema, haemorrhage, atrophy, infarction, mass effect, contrast enhancement / enhancing lesions, calcification, developmental malformations, hydrocephalus.

Results: $51.9 \%$ of study population (110) were males and $48.1 \%$ (102) were females. The mean age of study population was 39.5 with a standard deviation of 16.3.All patients were subjected to CT scan and 117 cases (55.2\%) had normal CT scan. 95 cases had abnormal CT findings. On MRI brain abnormality was detected in $50 \%$ of total cases. i.e. 106 out of 212 cases. In our study MRI of brain diagnosed glioma, multiple sclerosis and meningoencephalitis which could not be detected on CT. The most common abnormalities were infarction (28 cases), tuberculoma (21 cases), neurocysticercosis (16 cases). 130 patients presented with GTCS out of which 64 (49.2\%) cases had abnormal neuroimaging findings. 74 patients presented with focal seizure out of which 39 $(52.7 \%)$ cases had abnormality. 7 patients presented with myoclonic seizure out of which 3 (42.8\%) cases had abnormality. 1 case presenting as absence seizure had normal MRI. Out of 106abnormal MRI findings, 45 patients had vascular, 41 had infective 7 had neoplastic and 13 had other etiologies.

Conclusion: Neuroimaging has 50\% chance of finding any abnormality of brain in patients presenting with seizure. Hence it plays an important role in diagnosis and must be performed in all patients presenting with seizure.MRI plays an important role with its precise diagnosis like glioma and is more effective than CT scan in diagnosis of patients presenting with seizure. Patients presenting with focal seizure had the highest neuroimaging abnormality. In our study population of western Odisha vascular abnormalities were highest. In addition to it frequency of infective causes of seizure was also high in this area.
\end{abstract}




\section{Introduction}

A seizure is a transient occurrence of signs and symptoms due to abnormal excessive or synchronous electrical activity in the brain. It is a result of shift in the normal balance of excitation and inhibition within the CNS. Although a variety of factors influence the incidence and prevalence of seizures, $\sim 5-10 \%$ of the population will have at least one seizure, with the highest incidence occurring in early childhood and late adulthood. ${ }^{[1]}$ Epilepsy is said to be present when there is

- At least two unprovoked seizures occurring more than 24 hours apart;

- One unprovoked seizure and a probability of further seizures similar to the general recurrence risk (at least 60\%)after two unprovoked seizures, occurring over next 10 years;

- Diagnosis of an epilepsy syndrome. ${ }^{[2][3]}$

The incidence of epilepsy is approximately 0.3 to $0.5 \%$ and prevalence is estimated to be 5 to 10 persons per 1000. ${ }^{[4]}$ Globally an estimated 2.4 million people are diagnosed with epilepsy each year. ${ }^{[5]}$ An estimated 6- 10 million individuals live with active epilepsy in India. ${ }^{[6][7][8]}$.

In Indian medical practice assessment of patients presenting with seizure is a challenge for a physician in evaluating and treating them. In order to diagnose and find out the etiology of seizure many investigations are available now a days. These include CT scan, MRI, MR spectroscopy, MR venogram, Carotid angiogram, EEG. After introduction of neuroimaging in seizure, they have been a great boon both for diagnosis of cerebral lesions as well as clinical management of patients with neurological impairments. Neuroimaging is useful in the diagnosis of the abnormalities underlying the seizures. After the first unprovoked seizure, imaging in adults has a clinically significant yield of about $10 \%$, leading to the diagnosis of structural lesions. CT scans are generally the first modality used, since they aremore readily available. CT scans can accurately detect hemorrhage, infarctions, gross malformations, ventricular system pathologies and lesions with underlying calcification.CT scanning gives limited information on major problems that may underlie epilepsy but MRI is vastly superior in detecting the various structural causes of epilepsy. Nonetheless, CT may be the only feasible study when MRI is not available or in emergency circumstances.

MR imaging has emerged as the more diagnostically valuable and most valuable tool for preoperative localization of epileptogenic focus because of its excellent soft tissue contrast, allowing for detailed depiction of anatomy, freedom from beam - hardening artefact in basal brain that occur with $\mathrm{CT}$ and capacity for multiplanar imaging. ${ }^{[9]}$ MRI plays an essential role in every aspect of the diagnosis and management of seizures. The diagnosis of epilepsy with MRI has made this diagnostic tool beyond compare to other investigations. MRI is the appropriate neuroimaging study of choice.

This is beyond doubt that MRI is the most crucial procedure in the diagnosis, treatment and followup of patients of inflammatory and parasitic lesions of the brain such as neurocysticercosis, tuberculoma, brain abscess, and meningoencephalitis. MRI provides exact localization and histological nature of lesions.

Due to multiple challenges many patients in India presenting with seizure are difficult to evaluate properly to find the etiology of seizure. In The following study has been undertaken in western Odisha in a tertiary care hospital and an attempt has been made to study the etiology and spectrum of neuroimaging findings in patients with seizures.

\section{Aims and Objectives}

- To study the neuroimaging findings in patients presenting with seizure and to determine etiology.

- To correlate type of seizure with neuroimaging findings. 


\section{Materials and Methods}

A cross sectional study was conducted in department of internal medicine, VIMSAR from October 2016 to September 2018. Total 212 cases of age $>14$ years who presented with seizure were studied and patients having implants, pregnant and claustrophobic patients were excluded.

A detailed history was taken and clinical examination was done in the patients. The points noted were duration of illness, type of seizures, any associated illness. Detailed clinical and neurological examination was done to find any neurological deficit. Based on the history and examination, a clinicoetiological diagnosis was made.A detailed proforma was filled up and all the patients underwent routine investigations like complete blood count, serum urea and creatinine, serum electrolytes, LFT. All the patients were subjected to CT scan and MRI of brain by a 160 slice CT scan machine and 1.5 tesla MRI scanner respectively.

Neuroimaging findings were recorded in all patients as per the proforma. Every effort was made to make sure of high quality scans and to avoid artefacts. Various details of neuroimaging findings were studied like- Any lesion present, signal intensity/density, surrounding edema, haemorrhage, atrophy, infarction, mass effect, contrast enhancement / enhancing lesions, calcification, developmental malformations, hydrocephalus.

\section{Results}

$51.9 \%$ of population (110) were males and $48.1 \%$ (102) were females with a sex ratio of 1.07:1. Slight male predominance was recorded in this study population. The age range of patients was 70 with minimum age of 15 and maximum age of 85 . The mean age of study population was 39.5 with a standard deviation of 16.3. Maximum population $(24.1 \%)$ were in the age group of 31 40 years followed by 21-30 years (21.7\%). Minimum population $(0.9 \%)$ were in the age group of 81-90 years.
Fig-1 Distribution of study population according to age group

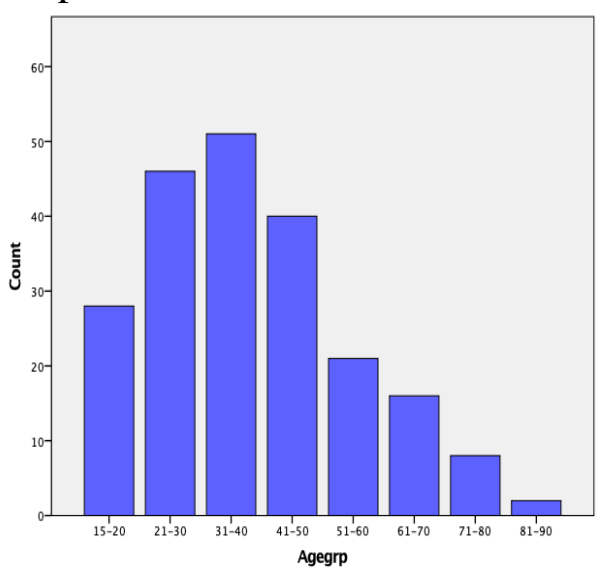

Out of 212 cases 130 (61.3\%) presented with GTCS, 74 (34.9\%) with focal seizure, 7 (3.3\%) with myoclonic seizure and $1(0.5 \%)$ with absence seizure. Out of all focal seizure cases $74 \%$ had intact awareness and $26 \%$ had impaired awareness.Focal seizure was more in females i.e. $62.2 \%$ focal seizure patients were females whereas GTCS was more in males i.e. $59.2 \%$ GTCS patients were males. Similarly, in myoclonic seizure patients $57.1 \%$ were males and $42.9 \%$ patients were females. 1 male patient had absence seizure.

All patients were subjected to CT scan and 117 cases $(55.2 \%)$ had normal CT scan. 95 cases had abnormal CT findings. 
Figure-2: CT Scan Findings in study Population

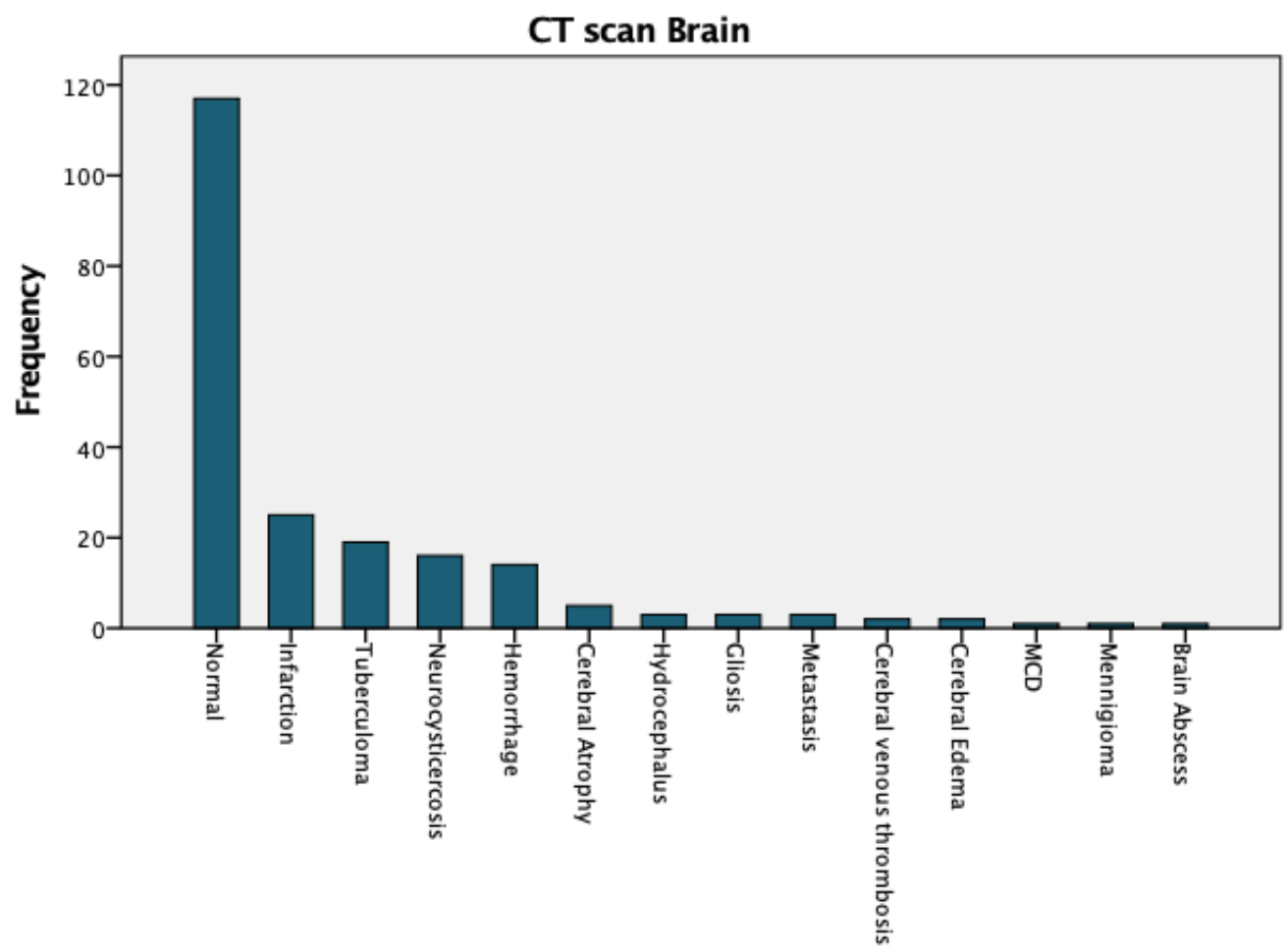

CT scan Brain

From CT scan Abnormality was found in $44.8 \%$ cases. Themost common abnormalities were infarction (25 cases) followed by Tuberculoma (19 cases) and neurocysticercosis (16 cases)

MRI of brain revealed precise picture in this study. It diagnosed some cases precisely which could not be diagnosed by CT scan. On MRI brain abnormality was detected in $50 \%$ of total cases. i.e. 106 out of 212 cases. In our study MRI of brain diagnosed glioma, multiple sclerosis and meningoencephalitis which could not be detected on CT. 1 case of glioma which was diagnosed as hydrocephalus on CT was confirmed on MRI. It also diagnosed some cases of infarction, tuberculoma, neurocysticercosis, cerebral venous thrombosis accurately.

Table-1: MRI Findings in Study Population

\begin{tabular}{|l|c|c|}
\hline MRI FINDINGS & FREQUENCY & PERCENTAGE \\
\hline Normal & 106 & $50 \%$ \\
\hline Infarction & 28 & $13.2 \%$ \\
\hline Hemorrhage & 14 & $6.6 \%$ \\
\hline $\begin{array}{l}\text { Cerebral venous } \\
\text { thrombosis }\end{array}$ & 3 & $1.4 \%$ \\
\hline Gliosis & 3 & $1.4 \%$ \\
\hline Neurocysticercosis & 16 & $7.5 \%$ \\
\hline Tuberculoma & 21 & $9.9 \%$ \\
\hline
\end{tabular}

\begin{tabular}{|l|c|l|}
\hline Brain abscess & 1 & $0.5 \%$ \\
\hline Meningoencephalitis & 3 & $1.4 \%$ \\
\hline Metastasis & 3 & $1.4 \%$ \\
\hline Meningioma & 1 & $0.5 \%$ \\
\hline Glioma & 3 & $1.4 \%$ \\
\hline MCD & 2 & $0.9 \%$ \\
\hline Hydrocephalus & 2 & $0.9 \%$ \\
\hline Cerebral atrophy & 5 & $2.4 \%$ \\
\hline Multiple Sclerosis & 1 & $0.5 \%$ \\
\hline TOTAL & 212 & $100 \%$ \\
\hline
\end{tabular}

The most common abnormalities were infarction (28 cases), tuberculoma (21 cases), neurocysticercosis (16 cases). The MRI findings with age group is tabulated in table-2 which shows maximum number of abnormality in 31-40 age group.

Out of 28 cases of infarction $16(57.1 \%)$ patients of were males and $12(42.9 \%)$ were females. Maximum cases ( 9 cases) were in the age group of 41-50. 7 cases were in the age group of 51-60, 6 cases were in age group of 61-70, 3 cases in 71-80 age group, 1 case in age group of 21-30 and 2 cases in age group of 31-40.

Total $21(9.9 \%)$ cases of tuberculoma were diagnosed in our study population. $13(61.9 \%)$ cases were males and $8(38.1 \%)$ cases were 
females. More number of cases were detected in young age groups. 6 cases in age group of 21-30, 5 cases in 31-40, 4 cases each in 15-20 and 41-50 age groups, 1 case each in 51-60 and 61-70 age groups.

16 cases (7.5\%) were diagnosed as neurocysticercosis. 15 (93.8\%) cases were females and 1 case $(6.3 \%)$ was male. Maximum cases (5) belonged to both age groups of 21-30 and 31-40. 3 cases were in age group of 15-20, 2 cases in 41-50 and 1 case in 51-60 age group.

Figure 3: Gender and MRI Findings

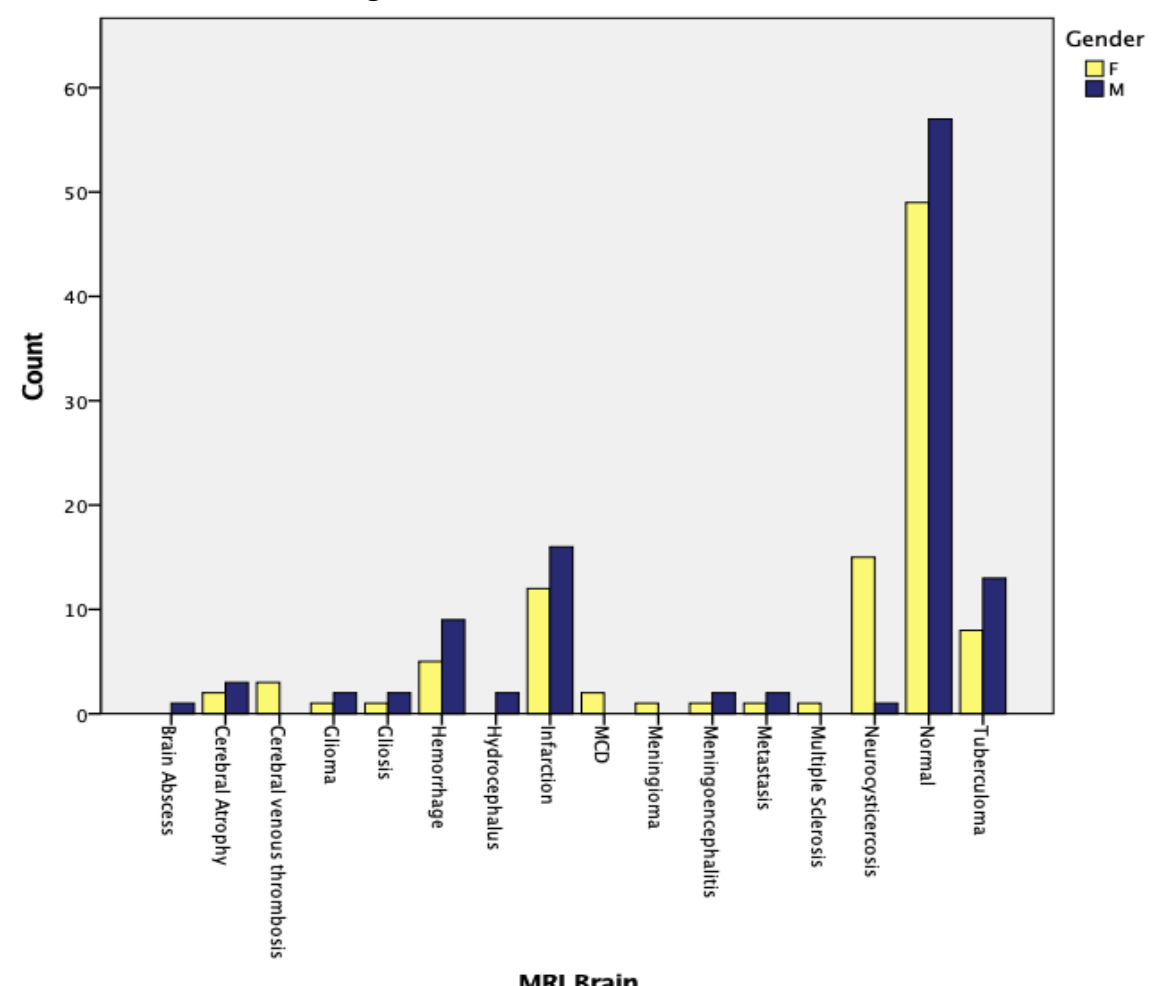

Table-2: Age Group and MRI Findings

\begin{tabular}{|l|c|c|c|c|c|c|c|c|c|c|}
\hline & \multicolumn{7}{|c|}{ Age group } & \\
\cline { 2 - 9 } & & $15-20$ & $21-30$ & $31-40$ & $41-50$ & $51-60$ & $61-70$ & $71-80$ & $81-90$ & Total \\
\hline MRI Brain & Brain Abscess & 1 & 0 & 0 & 0 & 0 & 0 & 0 & 0 & 1 \\
& Cerebral Atrophy & 0 & 0 & 0 & 0 & 4 & 0 & 0 & 1 & 5 \\
Cerebral venous thrombosis & 0 & 0 & 2 & 1 & 0 & 0 & 0 & 0 & 3 \\
Glioma & 1 & 0 & 1 & 1 & 0 & 0 & 0 & 0 & 3 \\
Gliosis & 0 & 0 & 2 & 0 & 0 & 0 & 1 & 0 & 3 \\
Hemorrhage & 0 & 0 & 1 & 1 & 3 & 6 & 2 & 1 & 14 \\
Hydrocephalus & 2 & 0 & 0 & 0 & 0 & 0 & 0 & 0 & 2 \\
Infarction & 0 & 1 & 2 & 9 & 7 & 6 & 3 & 0 & 28 \\
MCD & 2 & 0 & 0 & 0 & 0 & 0 & 0 & 0 & 2 \\
Meningioma & 0 & 1 & 0 & 0 & 0 & 0 & 0 & 0 & 1 \\
Meningoencephalitis & 0 & 0 & 1 & 1 & 0 & 1 & 0 & 0 & 3 \\
Metastasis & 0 & 0 & 2 & 1 & 0 & 0 & 0 & 0 & 3 \\
Multiple Sclerosis & 0 & 1 & 0 & 0 & 0 & 0 & 0 & 0 & 1 \\
Neurocysticercosis & 3 & 5 & 5 & 2 & 1 & 0 & 0 & 0 & 16 \\
Normal & 15 & 32 & 30 & 20 & 5 & 2 & 2 & 0 & 106 \\
Tuberculoma & 4 & 6 & 5 & 4 & 1 & 1 & 0 & 0 & 21 \\
Total & 28 & 46 & 51 & 40 & 21 & 16 & 8 & 2 & 212 \\
\hline
\end{tabular}

In our study 130 patients presented with GTCS out of which 64 (49.2\%) cases had abnormal neuroimaging findings. 74 patients presented with focal seizure out of which $39(52.7 \%)$ cases had 
abnormality. 7 patients presented with myoclonic seizure out of which $3(42.8 \%)$ cases had abnormality. 1 case presenting as absence seizure had normal MRI.

Among the 28 patients of infarction (the most common abnormality in our study) 17 cases presented as GTCS and 11 cases presented as focal seizure. Out of 21 patients of Tuberculoma 10 had GTCS, 10 had focal seizure and 1 had myoclonic seizure. Out of 16 cases of neurocysticercosis 8 patients presented as GTCS, 7 as focal seizure and 1 as myoclonic seizure.

Table-3: MRI Findings and Type of Seizure

\begin{tabular}{|c|c|c|c|c|c|c|}
\hline & \multicolumn{4}{|c|}{ Type of seizure } & \multirow[b]{2}{*}{ Total } \\
\hline & & Absence & Focal & GTCS & Myoclonic & \\
\hline \multirow[t]{16}{*}{ MRI Brain } & Brain Abscess & 0 & 1 & 0 & 0 & 1 \\
\hline & Cerebral Atrophy & 0 & 1 & 4 & 0 & 5 \\
\hline & Cerebral venous thrombosis & 0 & 2 & 1 & 0 & 3 \\
\hline & Glioma & 0 & 1 & 1 & 1 & 3 \\
\hline & Gliosis & 0 & 1 & 2 & 0 & 3 \\
\hline & Hemorrhage & 0 & 1 & 13 & 0 & 14 \\
\hline & Hydrocephalus & 0 & 0 & 2 & 0 & 2 \\
\hline & Infarction & 0 & 11 & 17 & 0 & 28 \\
\hline & MCD & 0 & 1 & 1 & 0 & 2 \\
\hline & Meningioma & 0 & 0 & 1 & 0 & 1 \\
\hline & Meningoencephalitis & 0 & 2 & 1 & 0 & 3 \\
\hline & Metastasis & 0 & 1 & 2 & 0 & 3 \\
\hline & Multiple Sclerosis & 0 & 0 & 1 & 0 & 1 \\
\hline & Neurocysticercosis & 0 & 7 & 8 & 1 & 16 \\
\hline & Normal & 1 & 35 & 66 & 4 & 106 \\
\hline & Tuberculoma & 0 & 10 & 10 & 1 & 21 \\
\hline Total & & 1 & 74 & 130 & 7 & 212 \\
\hline
\end{tabular}

Out of 212 patients 106 had abnormal MRI findings. 45 patients had vascular, 41 had infective 7 had neoplastic and 13 had other etiologies.

Figure 4: Neuroimaging Etiology in Study Population

\section{NEUROIMAGING ETIOLOGY}

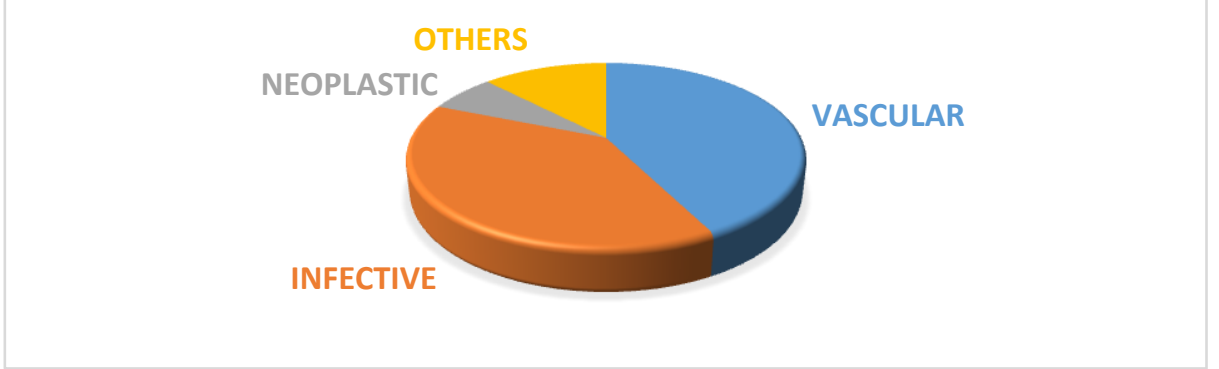

\section{Discussion}

Patients presenting with seizures have wide range of neuroimaging abnormalities depending upon the etiology. Neuroimaging can reliably identify and localize the intracranial abnormality so that further management can be executed accordingly. In our study 212 patients with clinical diagnosis of seizures were selected as per the criteria laid down by ILAE 2017 . The clinical history of each patient was recorded and al routine biochemical investigations were done as per proforma. CT scan was carried out with 160 slice CT scan machine and MRI was carried out with 1.5 Tesla MRI machine. 
The mean age of study population was 39.5 with a standard deviation of 16.3 . $51.9 \%$ of population were males and $48.1 \%$ were females with a sex ratio of $1.07: 1$.The most common abnormality was infarction ( 28 cases) followed by tuberculoma (21 cases), neurocysticercosis (16 cases). Vascular etiology was the highest and infective etiology was nearly equal to vascular etiology.
In our study $106(50 \%)$ out of 212 patients had normal neuroimaging findings. Ladurner, et al. ${ }^{[10]}$ found normal neuroimaging findings in about 46 $\%$ of the cases in their study in epilepsy and Daras, et al. ${ }^{[1]}$ in $37.4 \%$ of cases. Ashwin $\mathrm{T}$ et al. found $50 \%$ normal neuroimaging findings in their study. ${ }^{[12]}$

Table-4: Comparison with Other Studies

\begin{tabular}{|l|c|c|c|c|}
\hline $\begin{array}{l}\text { NEUROIMAGING } \\
\text { FINDINGS }\end{array}$ & $\begin{array}{c}\text { PRESENT } \\
\text { STUDY }(\%)\end{array}$ & $\begin{array}{c}\text { ASHWIN T } \\
\text { et al. }(\%)\end{array}$ & $\begin{array}{c}\text { SWAROOPA } \\
\text { et al. }(\%)\end{array}$ & $\begin{array}{c}\text { RAO et al. } \\
(\%)\end{array}$ \\
\hline INFARCTION & 13.2 & 14 & - & 5 \\
\hline HEMORRHAGE & 6.6 & 3 & - & - \\
\hline CVT & 1.4 & 4 & - & - \\
\hline GLIOSIS & 1.4 & - & 1.3 & - \\
\hline NCC & 7.5 & 4 & 8 & 27 \\
\hline TUBERCULOMA & 9.9 & 1 & 10.6 & 7 \\
\hline MENINGOENCEPHALITIS & 1.4 & 1 & - & - \\
\hline BRAIN ABSCESS & 0.5 & - & - & - \\
\hline METASTASIS & 1.4 & - & 0.7 & - \\
\hline MENINGIOMA & 0.5 & - & 1.3 & - \\
\hline GLIOMA & 0.9 & - & 2 & - \\
\hline MCD & 0.9 & - & - & - \\
\hline HYDROCEPHALUS & 0.9 & - & - & - \\
\hline CEREBRAL ATROPHY & 2.4 & - & - & - \\
\hline MULTIPLE SCLEROSIS & 0.5 & - & - & - \\
\hline CALCIFIED GRANULOMA & - & 8 & 16 & 15 \\
\hline
\end{tabular}

Ashwin $\mathrm{T}$ et al. conducted a similar study and in their study infarction was also the most common abnormality on neuroimaging. In our study 28 cases out of $212(13.2 \%)$ had infarction. In their study $14 \%$ study population had infarction. $9.9 \%$ cases had tuberculoma in our study but only $1 \%$ cases had tuberculoma in their study. $7.5 \%$ cases had NCC in our study whereas they had $4 \%$ cases of NCC. In their study $8 \%$ patient had calcified granuloma whereas in our study we did not find any specific calcified granuloma.

Swaroopa et al. observed calcified granuloma as the most common abnormality (16\%) conducted in a study among 150 patients presented with seizure in Telengana. ${ }^{[13]}$ The percentages of tuberculoma and NCC were more than our study, whereas they did not observe any vascular etiology like infarction or intracerebral haemorrhage.

Rao et al. conducted a similar study on 100 adult patients and observed $27 \%$ cases of NCC and it was the most common neuroimaging abnormality in their study followed by calcified granuloma $(15 \%)$ and tuberculoma $(7 \%) .{ }^{[14]}$

\section{Conclusion}

Assessment of the patient presenting with seizure disorder is a common problem in clinical practice. Neuroimaging plays a pivotal role in the evaluation of patients with seizures. Accurate diagnosis of the cause of seizure is crucial for finding an effective treatment. Neuroimaging has highly sensitive and specific in identifying the underlying pathology in seizures. Neuroimaging not only identifies specific epileptogenic substrates, but also determines specific treatment and predicts prognosis. Employing appropriate imaging protocols and reviewing the images in a systemic manner helps in the identification of subtle epileptogenic structural abnormalities. 
This study was carried out in 212 patients with clinical impression of seizures by subjecting them to CT and MRI scan to evaluate the spectrum of findings, various etiologic factors for seizures, and the most common imaging abnormality. In our study 106 patients (50\%) had abnormal neuroimaging. Infarction (13.2\%), Tuberculoma (9.9\%), Neurocysticercosis (7.5\%) and haemorrhage $(6.6 \%)$ constitute the common abnormalities. Other findings include cerebral atrophy (2.4\%), cerebral venous thrombosis, glioma, gliosis, meningoencephalitis and metastasis (1.4\% each), MCD and hydrocephalus ( $0.9 \%$ each), brain abscess, multiple sclerosis, and meningioma ( $0.5 \%$ each).

From our study we found that

1) Neuroimaging has $50 \%$ chance of finding any abnormality of brain in patients presenting with seizure. Hence it plays an important role in diagnosis and must be performed in all patients presenting with seizure.

2) MRI plays an important role with its precise diagnosis like glioma and is more effective than CT scan in diagnosis of patients presenting with seizure.

3) Patients presenting with focal seizure had the highest neuroimaging abnormality.

4) In our study population of western Odisha vascular abnormalities were highest. In addition to it frequency of infective causes of seizure was also high in this area.

\section{References}

1. Lowenstein DH. Seizures and Epilepsy, Kasper DL, Fauci AS, Hauser SL, Longo DL, Jameson JL, LoscalzoJ,eds. Harrison's Principle of Internal Medicine. $20^{\text {th }}$ ed. New York, NY: McGraw-Hill;2018:3050.

2. Abou-Khali BW, Gallagher MJ, Macdonald RL. Epilepsies, Daroff RB,Mazziotta JC, Jankovic J, Pomeroy SL,eds. Bradley's Neurology In Clinical Practice. $7^{\text {th }}$ ed. Philadelphia, PA: Elsevier/ Saunders;2016:1563.
3. Definition of Epilepsy 2014. Retrieved from https://www.ilae.org/guidelines/definitionand-classification/definition-of-epilepsy-2014

4. Goodridge DMG, Shorvon Sd. Epileptic seizures in a population of 6000 demography, diagnosis and classification and role of the hospital services. Br Med J 1983;287:641647.

5. World Health Organisation. (2016). Epilepsy (Fact sheet No. 999). Retrieved from http://www.who.int/mediacentre/factsheets/fs 999/en/

6. Tripathi M, Jain D, Devi M. Need for a national epilepsy control program. AnnIndian AcadNeurol2012;15:89-93

7. Devi G, Satishchandra P, Gururaj G. National workshop on public health aspects of epilepsy for senior personnel of state health departments in India. Ann Indian Acad Neurol1999;2:29-30.

8. Sridharan R, Murthy BN. Prevalence and pattern of epilepsy in India. Epilepsia 1999;40:631-636.

9. Scott N. Atlas. Magnetic Resonance Imaging of the brain and spine. $4^{\text {th }}$ edn., p. 2-14, 307339.

10. G. Ladurner, W.D. Sager. Summary: The value of $\mathrm{CT}$ and EEG in the diagnosis of epilepsy. Fortschr. Neurol. Psychiat., 1979; 47: 264-268.

11. Daras M, Tuchman AJ, Strobos RJ. Computed tomography in adult-onset epileptic seizures in a city hospital population. ClinExp Neurol., 1987; 24: 159-6

12. Ashwin T, Tumbanatham A, Green SR, Singh KJ. Clinico etiological profile of seizures in adults attending a tertiary care hospital. Int $\mathbf{J}$ Adv Med 2017;4:490-6.

13. Deme S. A study of correlation of CT scan brain and EEG in epilepsy. IAIM, 2016; 3(10): 55-61

14. Chalapathi Rao K.N.S.S.V, Siddnathi Narasinga Rao, Atluri Sridevi, M.S. Naga Pavan Kumar, Metta Srinivas, Madhuvulu Buchineni. "Clinical and Radiological 
Evaluation of New-Onset Epileptic Seizures

in a Tertiary Care Hospital". Journal of

Evidenced based Medicine and Healthcare;

Volume 2, Issue 39, September 28, 2015;

Page: 6452-6457, DOI:

$10.18410 / \mathrm{jebmh} / 2015 / 883$. 